\title{
Pineal Apoplexy: Imaging Diagnosis and Follow-up of Three New Cases
}

\author{
Archana Asundi, Donatella Tampieri, Denis Melançon, Rolando Del Maestro, Kevin Petrecca, \\ Maria delPilar Cortes
}

Can. J. Neurol. Sci. 2011; 38: 931-933

Cystic lesions on the pineal gland are a common phenomenon, being found on up to $40 \%$ of autopsy cases ${ }^{1}$. However, symptomatology as a result of these cysts is considerably rare. Mukherjee, Banerji and Sharma identified 74 cases in the literature ${ }^{1}$. Symptomatic pineal cysts present as one of three syndromes identified by Wisoff and Epstein²: (1) paroxysmal headache with gaze paresis, (2) chronic headache, gaze paresis, papilledema and hydrocephalus, and (3) pineal apoplexy with acute hydrocephalus. Pineal apoplexy is understood as an acute presentation of hemorrhage within a pineal cystic lesion.

Of the three syndromes pineal apoplexy is the rarest and while poorly understood, can have the most catastrophic of consequences, resulting in sudden death ${ }^{3}$. Herein we report three cases with clinical and imaging findings suggestive of pineal gland apoplexy in the context of a discussion of the relevant literature.

\section{Materials ANd Methods}

We conducted a retrospective review of the medical charts and imaging findings of three patients who presented to the Montreal Neurological Institute between 2008 and 2010 with symptoms and radiological findings corresponding to pineal apoplexy as identified by an enlarged pineal gland cyst with a fluid-fluid level suspicious for blood derivate content. We present an analysis of diagnostic imaging and follow-up studies in view of the relevant literature.

\section{RESUlTS}

Case 1. Figure 1A-G. A 42-year-old woman presented with a three-day history of sudden-onset severe headache. The magnetic resonance image (MRI) indicated non-communicating hydrocephalus. A $29 \mathrm{~mm}$ cystic lesion was noted protruding into the posterior third ventricle with a fluid-fluid level. Computed tomography (CT) scan demonstrated cystic hyperdense content

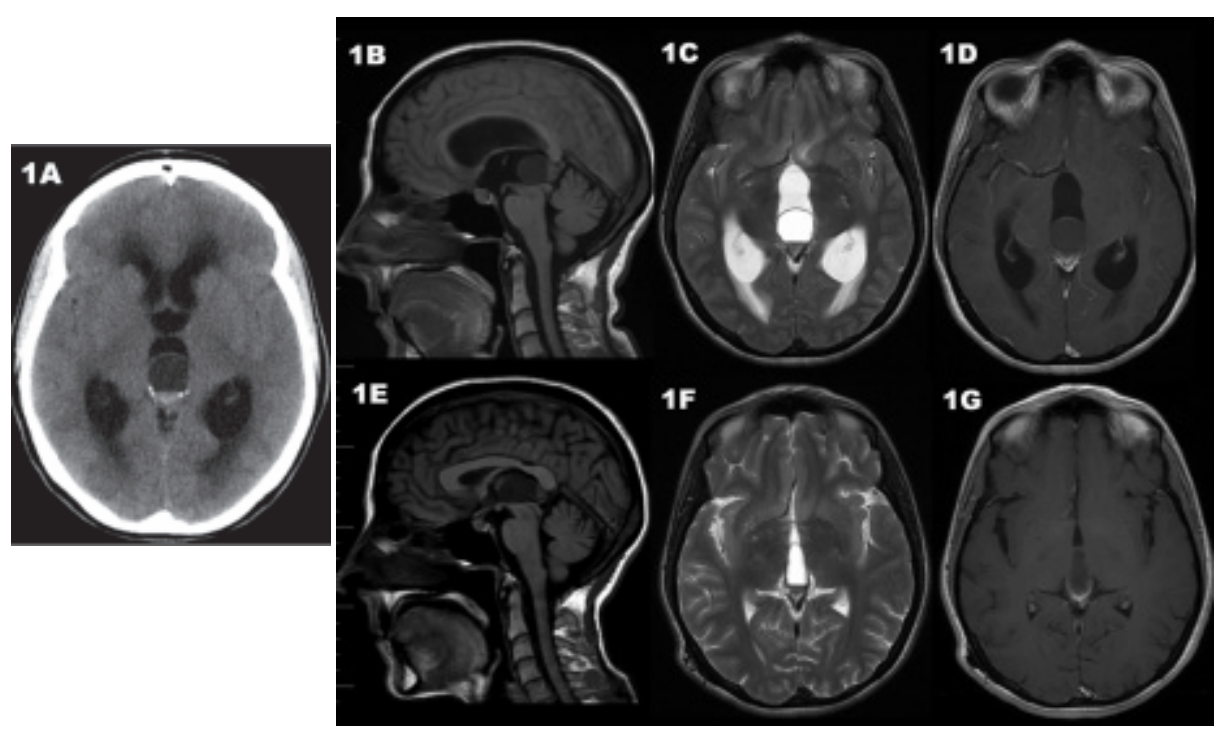

Figure 1: Case 1. Figure 1A. Axial CT Image with pineal cystic lesion protruding into the third ventricle. The lesion demonstrates hyperdense content with fluid level suggestive of intralesional hemorrhage. The image also shows diffuse hydrocephalus and transependymal exudates. Figures $1 B, 1 C, 1 D$. Sagittal T1-weighted (b), axial T2-weighted (c) and axial T1-weighted post-gadolinium $(d)$ MRI. The images demonstrate a pineal cystic lesion with a fluid-fluid level protruding into the third ventricle. Diffuse hydrocephalus is also present. The lesion shows thin wall enhancement with gadolinium (d). Figure $1 E$, $1 F, 1 G$. Sagittal T1-weighted (e), axial T2weighted $(f)$ and axial T1-weighted postgadolinium ( $g$ ) MRI at 14-month follow-up. There is complete resolution of the hydrocephalus with placement of the ventriculoperitoneal shunt. The appearance of the pineal lesion is unchanged with fluid-fluid level and thin peripheral enhancement $(g)$.

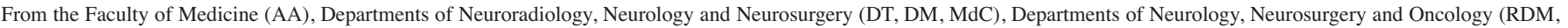
KP), Montreal Neurological Institute and Hospital, McGill University, Montreal, Quebec, Canada.

Received February 8, 2011. Final Revisions Submitted May 9, 2011.

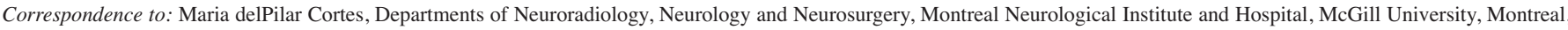
Quebec, Canada. 

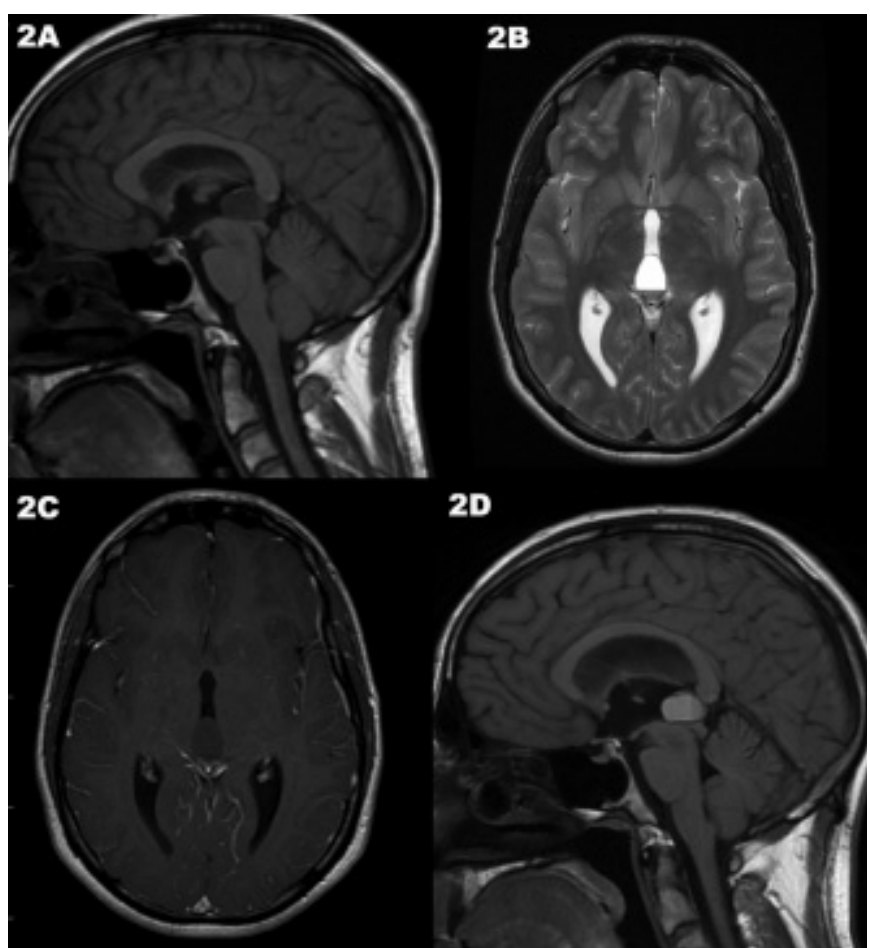

Figure 2: Case 2. Figure 2A, 2B, 2C. Sagittal T1-weighted (a), axial T2weighted (b) and axial T1-weighted (c) post-gadolinium MRI. Homogenous pineal lesion seen protruding into third ventricle. Lesion is cystic with fluid-fluid level and thin peripheral enhancement (c). Figure 2D. Sagittal T1-weighted MRI done at 24 months follow-up. MR image shows changes in the appearance with the now hyperintense lesion protruding into third ventricle and fluid-fluid level.

favoring intralesional hemorrhage. The lesion was mostly hypointense on T1-weighted images, hyperintense on T2weighted images and showed thin wall enhancement with gadolinium. A right ventriculoperitoneal shunt was inserted and the headache resolved. The overall size of the lesion slightly reduced after 14 months post-treatment and the patient remained asymptomatic.

Case 2. Figure 2A-D. A 30-year-old man presented with a two-month history of generalized headaches. A CT revealed a $21 \mathrm{~mm}$ pineal cystic lesion diameter with hyperdense fluid level protruding into the third ventricle. Magnetic resonance imaging showed that the lesion was responsible for focal mass effect with slight compression of the superior colliculi. The lesion showed a fluid-fluid level, isointensity on T1-weighted images, hyperintensity on T2-weighted images and thin peripheral enhancement. The MRI studies after 12 and 24 months showed a progressive increase in signal hyperintensity on T1-weighted images and stable T2 shortening.

Case 3. Figure 3A-B. A 36-year-old man with recurrent seizures was referred for a $12.5 \mathrm{~mm}$ pineal lesion impinging on the tectal plate and causing partial stenosis of the Sylvian aqueduct. The lesion was isointense on T1-weighted images and hyperintense on T2-weighted images with a fluid-fluid level. The lesion demonstrated thin peripheral enhancement. The MRI appearance at 19-month follow-up was unchanged.

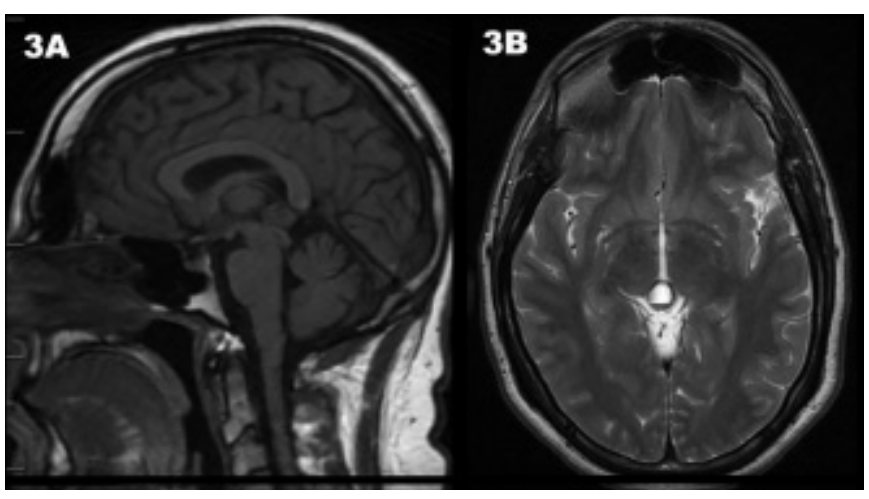

Figure 3: Case 3. Figure 3A, 3B. Sagittal T1-weighted and axial T2weighted magnetic resonance images at 19-month follow-up. MR image shows cystic appearance of the lesion and fluid-fluid level.

\section{Discussion}

Consistent with previously reported cases of pineal apoplexy, the main complaint for two out of three patients in this case report was severe headache. Michielsen et al determined three patterns of headache in patients with symptomatic pineal cysts: (1) sporadic headaches which have been occurring chronically, (2) paroxysmal headaches which only have a short-term history or (3) an acute prolonged headache ${ }^{4}$. The clinical presentation of our cases agrees with this classification; case 1 presented with an acute persistent headache and Case 2 with short-term paroxysmal headaches. The seizure disorder in Case 3 appears to be incidental, as there are no cases in the literature of pineal apoplexy presenting with epilepsy. Only in Case 1, however, was there obstructive hydrocephalus supporting Michielsen et al's claim that the presence of headache does not necessarily indicate hydrocephalus and may in fact be due to compression of pineal veins ${ }^{4}$. Visual symptoms are often identified in cases of pineal apoplexy, however this was not found in our series.

Pineal apoplexy has been considered an epiphenomenon ${ }^{2}$ that can occur in the presence of benign cysts or neoplastic processes. Pineal apoplexy can result in a wide range of imaging appearances thus complicating diagnosis of the underlying lesion, which is necessary to guide appropriate treatment.

On T1-weighted images, pineal cysts typically appear slightly hyperintense to cerebrospinal fluid (CSF) and hypointense to brain parenchyma. Pineal cysts appear strongly hyperintense to brain parenchyma and often CSF on T2-weighted images ${ }^{1,5,6}$. In addition, pineal cysts often show a thin (no greater than $2 \mathrm{~mm}$ ) rim of enhancement upon contrast due to surrounding pineal parenchyma, which lacks a blood-brain barrier ${ }^{5}$. Pineal tumors on the other hand, have been reported to typically appear similar to grey matter on MRIs ${ }^{5,6}$. In all our cases, appearances in CT and MRI were indicative of blood derivate content within the lesion, which obscured its appearance, hindering identification of the pathological process underlying the lesion. The MR signal appearance resembling blood products thus complicates diagnosis of the underlying lesion, as intensity signals are more suggestive of hemoglobin denaturation instead of high 
proteinaceous content. It is also of interest to note that at least one of our cases had a discreet change in the intensity of the signal in T1 sequence on the follow up imaging. This might represent a slower denaturation of hemoglobin when compared to parenchymal hemorrhagic lesions.

However, all cases in our series showed enhancement of a thin capsule which is strongly suggestive of the presence of a benign cyst. Further long-term follow-up should be done to assess whether lesions are temporally stable which may also support a benign process. Apart from benign cysts, the differential diagnosis should include neoplastic lesions such as pineal parenchymal tumors (e.g. pineocytomas and pineoblastomas), papillary tumors, gliomas and germ cell tumors (e.g. germinomas and teratomas). We believe histopathological analysis of a pineal lesion would conclusively identify a neoplastic process when there are changes in the imaging appearance over the follow up.

Given the lack of correlation between imaging appearance and symptoms, most literature suggests follow-up on the basis of clinical symptoms. It is generally agreed that asymptomatic pineal apoplexy does not predicate treatment but there is no consensus about the treatment of symptomatic cases. Surgical resection has been favored among cases in the literature, especially in cases showing rapid deterioration in symptoms. However, a report by Avery et al, documented a case treated successfully through a minimally invasive approach ${ }^{7}$. In our cases, conservative or minimally invasive treatment, in the case of a ventriculoperitoneal shunt, was successful. It should be noted that none of our patients presented with visual symptoms or rapid deterioration, which may be an indication for more invasive measures ${ }^{1}$. Considering that histopathological analysis may not be reliable ${ }^{2}$ due to the cystic nature of the lesion, surgical excision for merely diagnostic purposes may not be indicated. Therefore, conservative management or ventriculoperitoneal shunt insertion should be considered in the treatment of pineal apoplexy as it reduces the risk of complications from a more invasive surgical approach, since the lesions remain stable while untreated.

In conclusion, symptomatic pineal apoplexy is a rare condition, however, a report of fatal outcome should raise awareness of this entity ${ }^{3}$. Headache is characteristic of apoplexy, although highly non-specific and variable in presentation. Definite histopathological diagnosis of the underlying lesion is warranted to more conclusively exclude a neoplastic process when there is progression of the size or change in the appearance of the lesion. This was not obtained in our cases given the stability of the lesions. Finally, although surgical resection is mentioned in the literature as the treatment-of-choice, our cases had a successful outcome with conservative management or only ventricular derivation suggesting invasive surgical intervention may be unnecessary. Further research should continue to follow these patients for an extended time in order to assess whether pineal apoplexy in asymptomatic patients shows temporal stability and whether more conservatively managed patients continue to have positive outcomes.

\section{REFERENCES}

1. Mukherjee KK, Banerji D, Sharma R. Pineal cyst presenting with intracystic and subarachnoid haemorrhage: report of a case and review of the literature. Br J Neurosurg. 1999; 13(2): 189-92.

2. Wisoff JH, Epstein F. Surgical management of symptomatic pineal cysts. J Neurosurg. 1992; 77(6): 896-900.

3. Richardson JK, Hirsch CS. Sudden, unexpected death due to "pineal apoplexy". Am J Forensic Med Pathol, 1986; 7(1): 64-8.

4. Michielsen G, Benoit Y, Baert E, Meire F, Caemaert J. Symptomatic pineal cysts: clinical manifestations and management. Acta Neurochir (Wien). 2002; 144(3): 233-42; discussion 242.

5. Mamourian AC, Yarnell T. Enhancement of pineal cysts on MR images. AJNR Am J Neuroradiol. 1991; 12(4): 773-4.

6. Sarikaya-Seiwert S, Turowski B, Hanggi D, et al. Symptomatic intracystic hemorrhage in pineal cysts. Report of 3 cases. J Neurosurg Pediatr. 2009; 4(2): 130-6.

7. Avery GJ, Lind CR, Bok AP. Successful conservative operative management of pineal apoplexy. J Clin Neurosci. 2004; 11(6): $667-9$. 